\title{
Some Comparative Studies for Cursive Handwritten Tifinagh Characters Recognition Systems
}

\author{
B. EL Kessab, C. Daoui, B. Bouikhalene and R. Salouan \\ Laboratory of Information Processing and Decision Support, \\ Faculty of Science and Technology, BP 523, Beni Mellal, Morocco \\ bade10@hotmail.fr;daouic@yahoo.com,bbouikhalene@yahoo.fr, \\ rachidsalouan@gmail.com
}

\begin{abstract}
In this research, we present two comparative studies; the first one is between two methods of features extraction which are the mathematical morphology, the zoning and the hybridization of these two methods. The second comparative study is between both supervised methods used in learning-classification which are the Multi-Layer Perceptron (MLP) and the Support Vector Machines (SVM) applied to cursive handwritten Tifinagh characters recognition. The obtained experimental result demonstrates that the hybrid method is most efficient and the SVM is more performing than the MLP.
\end{abstract}

Keywords: The cursive handwritten Tifinagh characters, the thresholding, the centering and the normalization techniques, the zoning and the mathematical morphology methods, the Multi-Layer Perceptron (MLP), The Support Vectors Machines (SVM)

\section{Introduction}

Currently, handwritten character recognition is one of the most interesting fields of pattern recognition and artificial intelligence. It undoubtedly plays a very important role in the actual world and really can solve many complex problems in different fields such as bank cheques recognition, postal code recognition, etc. Several studies intended for handwritten character recognition in different languages have been developed using the structural methods in features extraction [19-25] and using the support vector machines [1-7, 26, 28] or the neural networks [8-18].

This paper focuses on cursive handwritten Tifinagh characters recognition systems. In fact, a succession of operations used in this recognition system which can be fragmented into three principal phases. The first one is the preprocessing which serves to clean the character image in order to enhance its quality including median filter, thresholding, normalization and centering techniques. The second phase is features extraction for avoiding data abundance, well as reducing its dimension that is to say the character image is converted to a vector, and in this context we have used the mathematical morphology, the zoning and the hybridization between them. The last phase is the learning-classification or recognition, we have opted the multi-layer perceptron and the support vector machines. In order to improve our recognition systems performances, we present two comparative studies; the first one is between two methods of features extraction which are the mathematical morphology, the zoning and the hybridization of these two methods. The second comparative study is between both supervised methods used in learning-classification which are the Multi-Layer Perceptron (MLP) and the Support Vector Machines (SVM).

This paper is organized as follows: The proposed system is given in Section 1. Preprocessing process is presented in Section 2. In Section 3 features extraction phase is 
described. Section 4 deals with the recognition phase. Experimental results are given in Section 5. Finally, this work is ended by a conclusion.

\section{Recognition System}

Our recognition system is presented as follow:

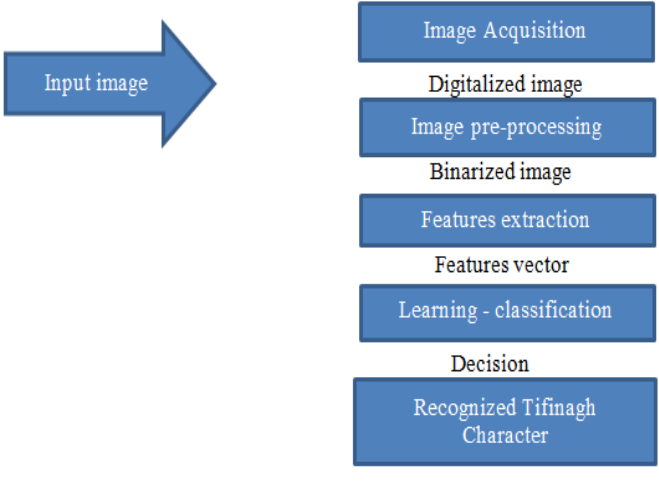

Figure 1. The Proposed Recognition System

\subsection{Tifinagh Character Database}

The used database contains Tifinagh cursive handwritten characters. Each character is written by many different scripters. An example of this database is presented in Figure 2.

\begin{tabular}{|c|c|c|c|c|c|}
\hline Cursive characters & Scripter 1 & Scripter 2 & Scripter 3 & Scripter 4 & Scripter 5 \\
\hline$\Lambda$ & & & 11 & & \\
\hline$\downarrow$ & & $i$ & $y$ & & \\
\hline$\square$ & & & & & L \\
\hline & & & & $<$ & 5 \\
\hline & & & & & ( \\
\hline$X$ & 5 & $1-2$ & $c$ & & 3 \\
\hline
\end{tabular}

Figure 2. Example of Cursive Handwritten Tifinagh Characters

\subsection{Pre-processing}

The pre-processing is a very important step in pattern recognition. It's the first phase of a recognition system used to produce a cleaned up version of the original image so that it can be used efficiently in the following phase that's the feature extraction. In this paper, we have pre-processed each character image by a median filter exploited for removing each noise from image. Then the thresholding is applied to render each image containing only the black and white colors according a preset threshold and after the centering technique is employed to position the character just in the center of its image. Finally we have used the normalization technique in order to normalize all sizes of character image. 


\subsection{Features Extraction}

This phase is exploited in order to extract from each character image its primitives which are the real values used as a components of a vector, it serves therefore to perform a vectorization of each character image which allows making easy the next phase.

In fact, several methods [19-25] can be exploited in this stage. In our recognition systems, we have used the mathematical morphology, the zoning and a hybridization method between them. Our goal is to ameliorate the performance of the proposed systems. Also we presented a comparison of theirs performances.

2.3.1. Extraction by Zoning Method: This method [21-24] can be explained as follow:

At first, given a black image containing a Tifinagh character that written in white. The zoning method consists to subdivide this image to several square or rectangular blocks or zones, then to count in each zone the number of white pixels. As consequence, the image is converted to a vector having a number of components equal to the number of zones (see Figure 3).

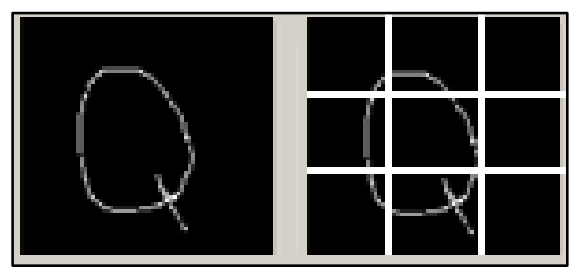

Figure 3. Features Extraction by Zoning Method from the Tifinagh Character

2.3.2. Extraction by Mathematical Morphology Method: The feature extraction is based on mathematical morphology [17-20]. The characteristic areas can be detected by the dilatation operation of the character image pre-processed in four directions. The characteristic zones can be detected by the intersections of dilations found to East, West, North and South.

Each point belongs to the characteristic area if and only if:

- This point does not belong to the limit of the object.

- From this point, moving in a straight line to the South, North, East and West we cross the object. The result of the extraction is illustrated in Figure 4.

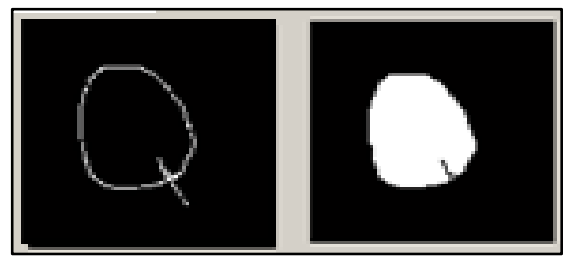

Figure 4. Features Extraction by Mathematical Morphology Method from the Tifinagh Character

2.3.3. Extraction by Hybrid Method: Mathematical Morphology + Zoning: This method consists after the features extraction by mathematical morphology to zoning it. But it is not like to that we carried previously, in fact, it comes this once around to achieve a zoning of the image by a zigzagged manner. In other words the zones in which the image is divided are a horizontal and vertical rectangles and a trapezoids which parallel to diagonal and also anti diagonal of the image. Then we will count the number of all white pixels in each of these zones in order to gather all these numbers in a vector (see Figure 5). 


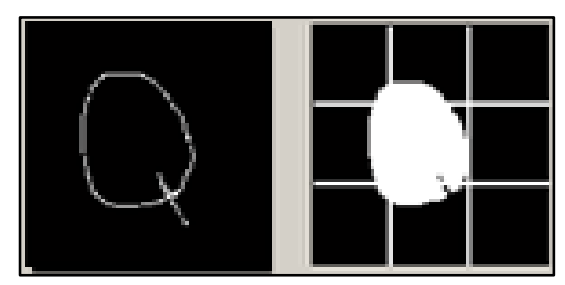

Figure 5. Features Extraction by all Method Used from Tifinagh Character

\section{Learning-classification Phase}

\subsection{The Neural Networks (NNs)}

The Neural Network [8-18] used in our work is a multi-layer perceptron (MLP) (see Figure $6)$.

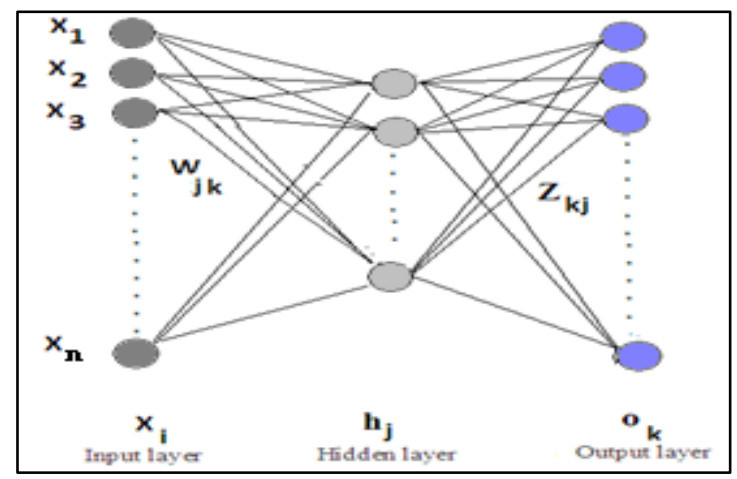

Figure 6. The Multi-layer Perceptron

The MLP is composed from the following elements:

- An input layer of $N$ vectors, each vector has $M$ components (features vector: $\mathrm{X}_{\mathrm{i}}$ ).

- A hidden layer of $\mathrm{P}$ activations neural $\mathrm{h}_{\mathrm{j}}$.

- An output layer of $\mathrm{N}$ activations neural $\mathrm{O}_{\mathrm{k}}$

- $\mathrm{N} \times \mathrm{P}$ connections between input layer and hidden layer, each weighted by $\mathrm{W}_{\mathrm{jk}}$.

- $\mathrm{P} \times \mathrm{N}$ connections between hidden layer and output layers, each weighted by $\mathrm{Z}_{\mathrm{kj}}$

Moreover, the operation of perceptron multi-layer learning is realized in five steps of back propagation algorithm:

- Step 1: (random Initialization of connexion weights $\mathrm{W}$ and $\mathrm{Z}$ ).

- Step 2: (propagation of input vectors of MLP):

Presentation of the inputs $X_{i}$ to input layer then propagation of these ones to hidden layer:

$$
h_{j}=f\left(\sum_{\mathrm{i}=1}^{\mathrm{n}} \mathrm{x}_{\mathrm{i}} \mathrm{w}_{\mathrm{ij}}\right)
$$

After from hidden layer to output layer

$$
Z_{k}=f\left(\sum_{j=1}^{n} h_{j} z_{k j}\right)
$$

With n: the number of hidden layer neurons.

Where $\mathrm{f}$ is called the activation function which is the sigmoid or logistic function:

$$
\mathrm{f}(\mathrm{x})=\frac{1}{1+e^{-x}}
$$


- Step 3: (calculation of error back propagation)

$$
E_{k}=O_{k}\left(1-O_{k}\right)\left(S_{k}-O_{k}\right)
$$

Where $S_{k}$ is the desired output (identity matrix) and $O_{K}$ is the real output (supervised learning).

Next, propagation of this error on the hidden layer; the error of each neuron of the hidden layer is:

$$
F_{j}=O_{k}\left(1-O_{k}\right) \sum_{k=1}^{n} Z_{k j} E_{k}
$$

- Step 4: (Correction of connections weights):

Afterward, change of the connection weights:

- Between input layer and hidden layer:

$$
\Delta W_{j i}=\alpha X_{i} F_{j}
$$

- Then between hidden layer and output layer:

$$
\Delta Z_{k j}=\alpha Y_{j} E_{k}
$$

Where $\alpha$ is the learning rate which is selected between 0 and 1 .

- Step 5:

After the learning of MLP. Using the Euclidean distance for classifying the test character.

$$
d\left(S_{k j}, O_{k j}\right)=\left(\sum_{i=1}^{n}\left(S_{k j}-O_{k j}\right)^{2}\right)^{1 / 2}
$$

The recognition will be attributed to the character that is very nearest to test character.

\subsection{The Supports Vectors Machines}

Support Vector Machines (SVM) are modern learning machines introduced by Vapnik [1], the principle of its functioning can be explained as follow:

For a two-class classification problem into space $\mathbb{R}^{\mathrm{p}}$, the first one contains a set of input vectors $\mathrm{x}_{1}, \mathrm{x}_{2}, \ldots, \mathrm{x}_{\mathrm{k}}$ with corresponding label $\mathrm{y}_{1}=1$, and the second class includes the vectors $\mathrm{x}_{\mathrm{k}+1}, \mathrm{x}_{\mathrm{k}+2}, \ldots, \mathrm{x}_{\mathrm{n}}$ and labeled by $\mathrm{y}_{2}=-1$.

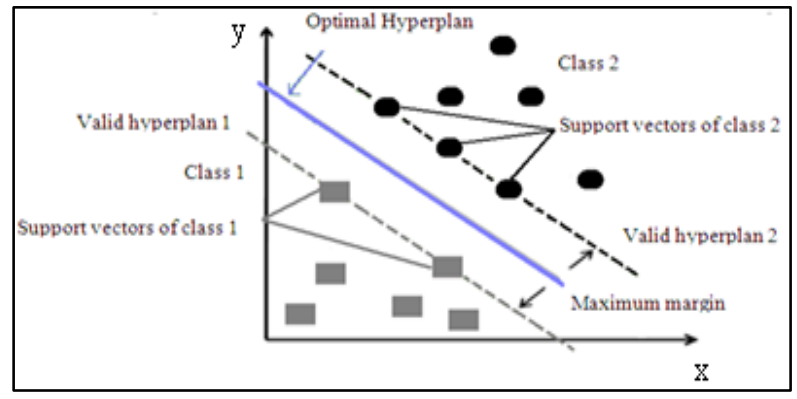

\section{Figure 7. The Support Vectors Machines Illustration}

The SVM consists to separate in an optimal manner between these both classes by mapping these vectors into a high dimensional feature space $\varphi\left(x_{i}\right) \in H(i=1,2 \ldots . . n)$. This separation is carried by a construction of an optimal hyper plane which maximizes as much the distance between the hyper plane and the nearest vectors of each class in the space $\mathrm{H}$. 
The mapping $\varphi$ (.) is realized by a special type of functions called the kernel functions $\mathrm{K}$ $\left(\mathrm{x}_{\mathrm{i}}, \mathrm{x}_{\mathrm{j}}\right)$ which defines an inner product in the space $\mathrm{H}$. Finally the decision function implemented by SVM can be written as:

$$
\mathrm{f}(\mathrm{x})=\operatorname{sign}\left(\sum_{i=1}^{n} \alpha_{i}^{*} y_{i} K\left(x, x_{i}\right)+b\right)
$$

Where $b$ is the offset of the optimal hyper plane from the origin, and the coefficients $\alpha_{i}$ are obtained by solving the convex quadratic programming problem.

Some example of the kernel functions:

\begin{tabular}{|l|l|}
\hline Kernel linear & $\mathrm{xy}$ \\
\hline Kernel polynomial of degree $\mathrm{n}$ & $(a x y+b)^{n}$ \\
\hline $\begin{array}{l}\text { Gaussian radial basis function (GRBF) of } \\
\text { a standard deviation } \sigma:\end{array}$ & $e^{-\frac{\|x-y\|^{2}}{2 \sigma^{2}}}$ \\
\hline
\end{tabular}

The method described above is designed for a problem of two classes only, many studies treat a generalization of the SVM to N classes [5, 27]. Among these studies, we have used in this work the strategy of one against all that is based to use $\mathrm{N}$ decision functions allowing to make a discrimination of a class bearing a label equal to 1 and containing a one vector against all other vectors included in a other class opposite that is labelled by the value -1 .

In the classification phase, we calculate the value image of an unknown vector $\mathrm{X}$ (test character) by all $\mathrm{N}$ decision functions that are obtained in the learning phase. The recognition will be attributed to the character that the decision function separates its class to another class containing the rest of characters which gives the biggest value.

\section{Experiments and Results}

$$
\text { Classe }(\mathrm{X})=\arg \max _{\mathrm{i}=1,2, \ldots, \mathrm{N}}\left(\mathrm{f}_{\mathrm{i}}(\mathrm{X})\right)
$$

In this work we want to compare between the performances of different extraction methods that are:

- Zoning.

- Mathematical morphology.

- Mathematical morphology + zoning.

When using the MLP then the SVM, that is to say to realize a second comparison between the performances of these methods of learning-classification.

In this research, each Tifinagh character is converted to some vector having:

- 9 components after using the mathematical morphology method.

- 9 components after using the zoning method.

\subsection{Recognition using MLP}

In order to implement this recognition system, we have opted the following data:

- A learning rate equal to 0.95 .

- A variable number of hidden layer neurons into $\{8,9,10,11,12\}$ just for knowing the effect of this variation on the performances of MLP. 
The obtained results of the recognition rates of each character $\boldsymbol{\tau}_{\mathbf{c}}$ and the global rate $\boldsymbol{\tau}_{\mathrm{g}}$ are grouped in the following table:

Table 1. The Recognition Rates $T_{c}$ and $T_{g}$ in Function of the Number of Hidden Layer Neurons by Using the Mathematical Morphology Method and the Mathematical Morphology + the Zoning Methods

\begin{tabular}{|c|c|c|c|c|c|c|c|c|c|c|c|}
\hline \multirow{3}{*}{\multicolumn{2}{|c|}{$\begin{array}{c}\text { Tifinagh } \\
\text { Characters }\end{array}$}} & \multicolumn{9}{|c|}{ MLP } & \\
\hline & & \multicolumn{5}{|c|}{$\tau_{\mathrm{c}}$ obtained by the mathematical morphology } & \multicolumn{5}{|c|}{$\begin{array}{c}\tau_{\mathrm{c}} \text { obtained by the mathematical morph logy }+ \text { the } \\
\text { zoning }\end{array}$} \\
\hline & & \multicolumn{5}{|c|}{ Number of hidden layer neurons } & \multicolumn{5}{|c|}{ Number of hidden layer neurois } \\
\hline & & \multirow{2}{*}{$\frac{8}{90.00}$} & \multirow{2}{*}{$\frac{9}{85.00}$} & \multirow{2}{*}{$\frac{10}{80.00}$} & \multirow{2}{*}{$\frac{11}{85.00}$} & \multirow{2}{*}{$\frac{12}{65.00}$} & \multirow{2}{*}{$\frac{8}{90.00}$} & \multirow{2}{*}{$\begin{array}{c}9 \\
90.00\end{array}$} & \multirow{2}{*}{$\frac{10}{90.00}$} & \multirow{2}{*}{$\frac{11}{100.00}$} & \multirow{2}{*}{$\begin{array}{c}12 \\
100.0\end{array}$} \\
\hline 1 & & & & & & & & & & & \\
\hline 2 & & 80.00 & 70.00 & 100.00 & 95.00 & 100.0 & 95.00 & 100.0 & 95.00 & 100.00 & 100.0 \\
\hline 3 & & 80.00 & 65.00 & 80.00 & 65.00 & 65.00 & 60.00 & 60.00 & 80.00 & 60.00 & 60.00 \\
\hline 4 & & 70.00 & 40.00 & 15.00 & 60.00 & 25.00 & 10.00 & 10.00 & 20.00 & 25.00 & 15.00 \\
\hline 5 & & 70.00 & 80.00 & 80.00 & 75.00 & 50.00 & 100.0 & 100.0 & 100.0 & 90.00 & 100.0 \\
\hline 6 & & 85.00 & 85.00 & 85.00 & 85.00 & 85.00 & 70.00 & 85.00 & 85.00 & 75.00 & 70.00 \\
\hline 7 & & 95.00 & 95.00 & 95.00 & 80.00 & 90.00 & 50.00 & 75.00 & 90.00 & 90.00 & 90.00 \\
\hline 8 & & 95.00 & 95.00 & 85.00 & 95.00 & 95.00 & 95.00 & 95.00 & 95.00 & 95.00 & 95.00 \\
\hline 9 & Y & 80.00 & 45.00 & 40.00 & 25.00 & 60.00 & 10.00 & 90.00 & 50.00 & 45.00 & 25.00 \\
\hline 10 & & 35.00 & 35.00 & 35.00 & 40.00 & 35.00 & 40.00 & 40.00 & 40.00 & 50.00 & 45.00 \\
\hline 11 & & 45.00 & 50.00 & 35.00 & 45.00 & 45.00 & 45.00 & 40.00 & 35.00 & 45.00 & 70.00 \\
\hline 12 & 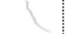 & 100.00 & 100.00 & 100.00 & 100.00 & 100.0 & 100.0 & 100.0 & 100.0 & 100.00 & 100.0 \\
\hline 13 & 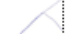 & 95.00 & 90.00 & 95.00 & 95.00 & 90.00 & 65.00 & 95.00 & 95.00 & 95.00 & 95.00 \\
\hline 14 & F & 60.00 & 60.00 & 55.00 & 60.00 & 55.00 & 70.00 & 60.00 & 65.00 & 60.00 & 60.00 \\
\hline 15 & 0 & 95.00 & 95.00 & 95.00 & 95.00 & 95.00 & 95.00 & 95.00 & 95.00 & 95.00 & 95.00 \\
\hline & $\tau_{g}$ & 73.44 & 62.75 & 67.20 & 68.75 & 65.94 & 62.20 & 70.94 & 65.56 & 70.32 & 75.63 \\
\hline
\end{tabular}

The associated graphs to the above table are:

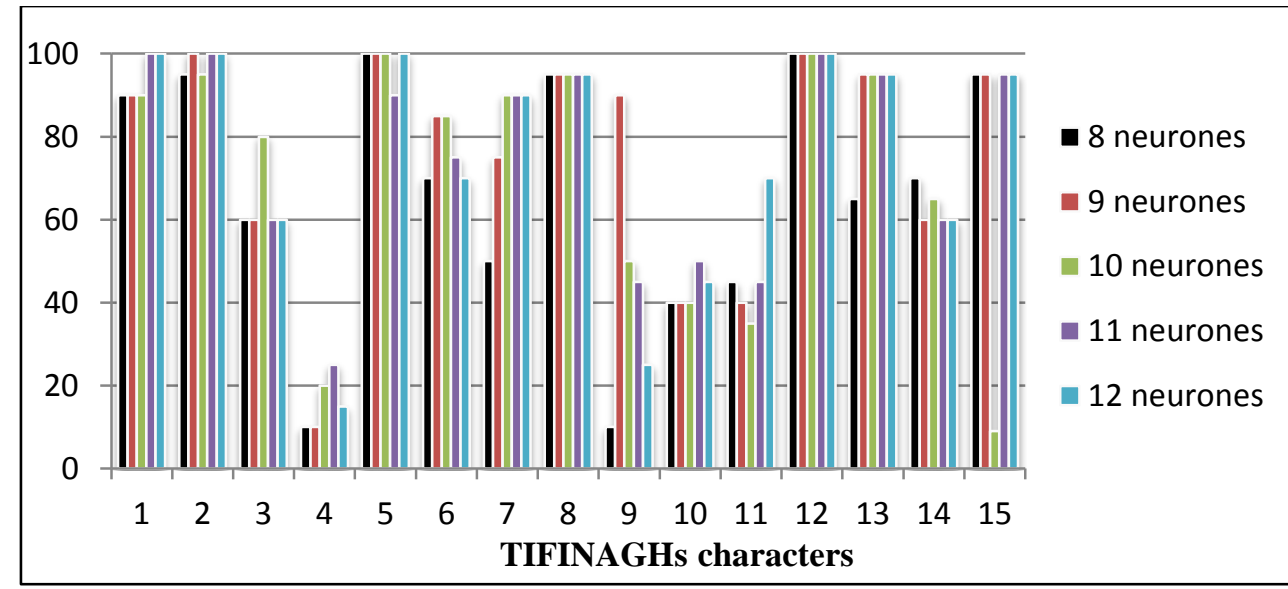

Figure 8. The Recognition Rate $\mathrm{T}_{\mathrm{c}}$ of each Tifinagh Character in Function of Hidden Layer Neurons by Using the Mathematical Morphology Method 


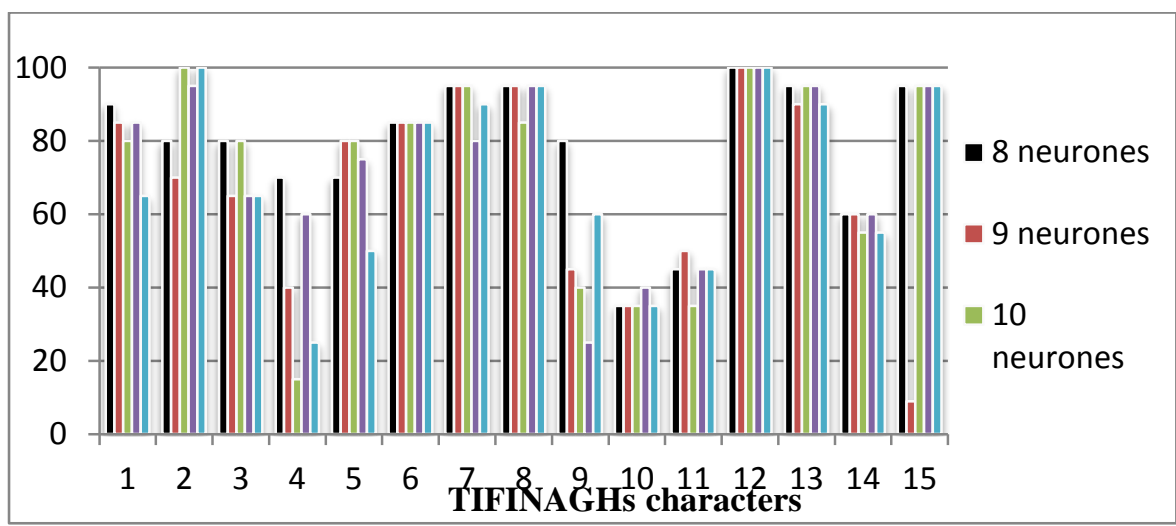

Figure 9. The Recognition Rate $\mathrm{T}_{\mathrm{c}}$ of each Tifinagh Character in Function of Number of Hidden Layer Neurons by Using the Mathematical Morphology and the Zoning Methods

- Analysis and comments:

After analysing the obtained recognition rates, we deduce that the hybrid method of mathematical morphology + zoning is more performing than that based only on mathematical morphology. Moreover, increasing the number of neurons in the hidden layer does not necessarily mean an increasing in rates.

\subsection{Recognition Using the SVM}

To realize a recognition system using the SVM, we have used the GBRF as a kernel function with a standard deviation $\sigma=0,9$.

Table 2. The Recognition Rates $T_{c}$ and $T_{g}$ which are Given in \% by Using the Zoning, the Mathematical Morphology and the Mathematical Morphology + the Zoning and the SVM

\begin{tabular}{|c|c|c|c|c|}
\hline \multirow{2}{*}{\multicolumn{2}{|c|}{$\begin{array}{c}\text { Tifinagh } \\
\text { Characters }\end{array}$}} & \multicolumn{3}{|c|}{ SVM } \\
\hline & & $\begin{array}{c}\tau_{\mathrm{c}} \text { obtained by the } \\
\text { zoning }\end{array}$ & $\begin{array}{c}\tau_{\mathrm{c}} \text { obtained by the } \\
\text { mathematical morphology }\end{array}$ & $\begin{array}{c}\tau_{\mathrm{c}} \text { obtained by the mathematical } \\
\text { morphology }+ \text { the zoning }\end{array}$ \\
\hline 1 & & 60.00 & 56.00 & 80.00 \\
\hline 2 & 1 & 65.00 & 90.00 & 70.00 \\
\hline 3 & & 55.00 & 58.00 & 60.00 \\
\hline 4 & & 60.00 & 56.00 & 54.00 \\
\hline 5 & & 50.00 & 54.00 & 50.00 \\
\hline 6 & & 50.00 & 50.00 & 55.00 \\
\hline 7 & & 70.00 & 55.00 & 80.00 \\
\hline 8 & 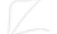 & 90.00 & 62.00 & 90.00 \\
\hline 9 & 1 & 70.00 & 51.00 & 98.00 \\
\hline 10 & 2 & 70.00 & 50.00 & 95.00 \\
\hline 11 & 3 & 50.00 & 40.00 & 56.00 \\
\hline 12 & 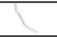 & 50.00 & 45.00 & 60.00 \\
\hline 13 & 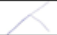 & 80.00 & 92.31 & 93.00 \\
\hline 14 & & 90.00 & 62.00 & 92.00 \\
\hline 15 & 0 & 60.00 & 60.00 & 60.00 \\
\hline & & 63.33 & 58.75 & 72.86 \\
\hline
\end{tabular}


The associated graphs to the above table are:

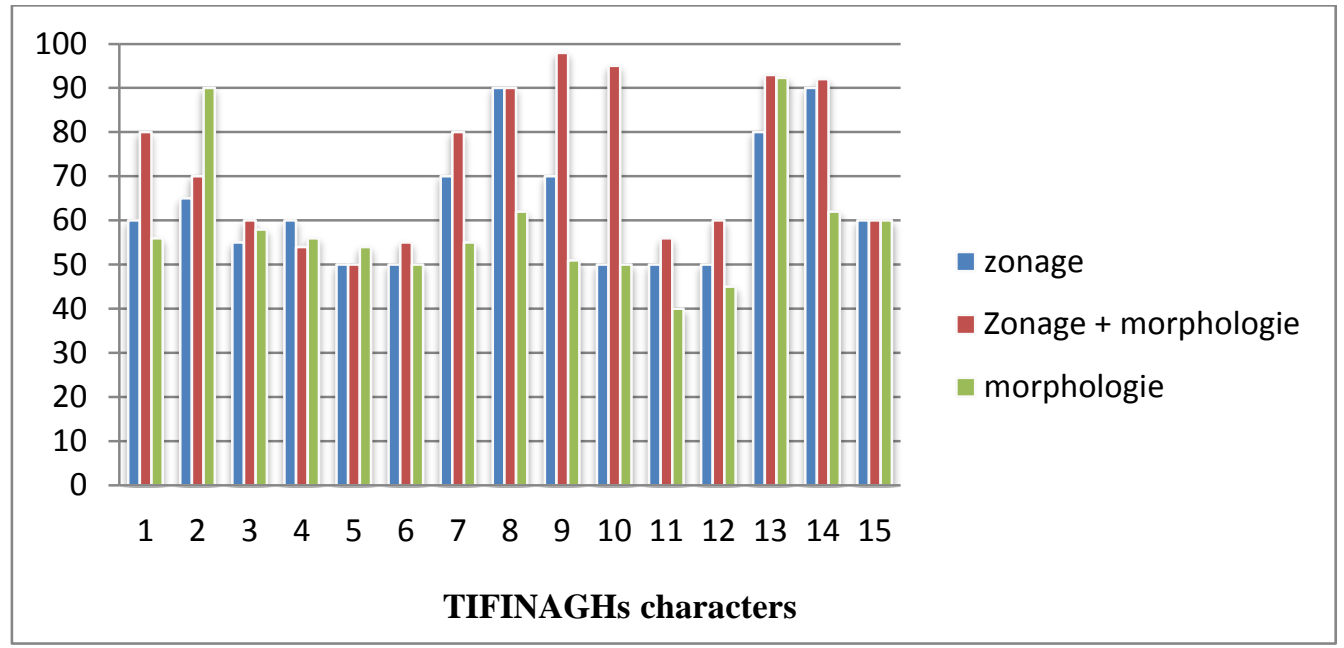

Figure 10. The Recognition Rate $\mathrm{T}_{\mathrm{c}}$ of each Tifinagh Character by Using the Mathematical Morphology, the Zoning Methods and the SVM

- Analysis and Comments:

Taking into account the results obtained after having implemented this recognition system, we can effectively conclude that the hybrid method Mathematical Morphology + Zoning is the most efficient followed by the Zoning then the Mathematical Morphology.

Finally, to schematize these two recognition systems, we present the following graphical interface that includes significantly all recognition phases.

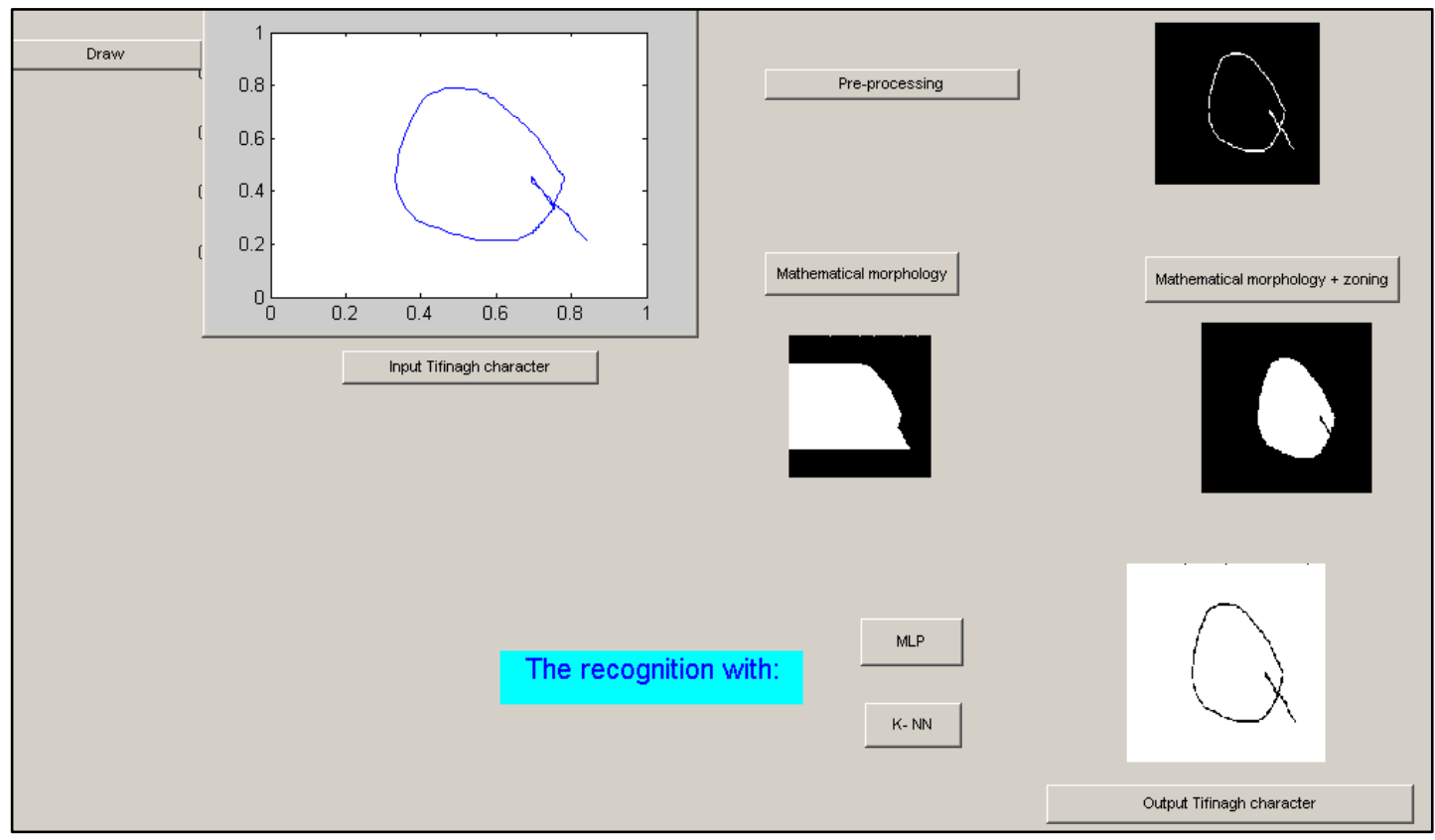

Figure 11. Implemented Graphical Interface to Recognize the Cursive Tifinagh Characters 


\section{Conclusion}

In this paper, we have presented two comparative studies for recognition of isolated cursive handwritten Tifinagh characters, the first one is carried between some methods of features extractions that are the mathematical morphology, the zoning and the hybridization between them. While the second comparison is realized between two methods of learningclassification which are the multi-layer perceptron and the support vector machines. For both studies we have used in the pre-processing phase the median filter, the thresholding, the normalization and the cantering techniques. The simulation result demonstrates that the hybrid method morphology + zoning is that the most performing followed by the Zoning then the Morphology in the features extraction and that the SVM is more efficient than the MLP.

\section{Acknowledgements}

We thank all those who assisted us to realize this research, in particular those who have contributed to construct the database of cursive handwritten Tifinagh characters.

\section{References}

[1] V. Vapnik. Support-vector networks. Machine Learning 20(3): 273, 1995.

[2] M. M. Adankon and M. Cheriet Model selection for the LS-SVM. Application to handwriting. Pattern Recognition, Volume 42, Issue 12, December 2009, Pages 3264-3270.

[3] F. Camastra A SVM-based cursive character. Pattern Recognition, Volume 40, Issue 12, December 2007, Pages 3721-3727.

[4] H. Drucker, D. Wu, and V. Vapnik. Support vector machines for spam categorization. IEEE transactions on Neural Networks, 10 5, 1999, pp. 1048-1055.

[5] C. Hou, F. Nie, C. Zhang, D. Yi, and Y. Wu Multiple rank multi-linear SVM for matrix data classification. Pattern Recognition, Volume 47, Issue 1, January 2014, Pages 454-469.

[6] J. John, K.V. Pramod, and K. Balakrishnan Unconstrained Handwritten Malayan Character Recognition using Wavelet Transform and Support vector Machine. Procedia Engineering, Volume30, 2012, Pages598-605.

[7] X. Niu and C. Y. Suen A novel hybrid CNN-SVM classifier for recognizing handwritten digits. Pattern Recognition, Volume 45, Issue 4, April 2012, Pages 1318-1325.

[8] S. Alma'adeed. Recognition of Off-Line Handwritten Arabic Words Using Neural Network, proc. of the Geometric Modeling and Imaging - New Trends, 2006.

[9] A. A. Desai. Gujarati handwritten numeral optical character reorganization through neural network. Pattern Recognition 43 (2010) 2582-2589.

[10] L. M. Fu Analysis of the dimensionality of neural networks for pattern recognition. Pattern Recognition, Volume 23, Issue10, 1990, Pages 1131-1140.

[11] K. Fukushima. Recognition of partly occluded patterns: A neural network model. Biol. Cyber net. vol. 84, (2001), pp. 251-259.

[12] A. Hennig and N. Sherkat Exploiting zoning based on approximating splines in cursive script recognition. Pattern Recognition, Volume 35, Issue 2, February 2002, Pages 445-454.

[13] P. Melin Modular Neural Networks and Type-2 Fuzzy Systems for Pattern Recognition. Springer Volume 3892012

[14] P. Nagare. License Plate Character Recognition System using Neural Network. International Journal of Computer Applications, Volume 25, No. 10, July 2011, pp. 36-39.

[15] I.S. Oh and C.Y. Suen. A class-modular feed-forward neural network for handwriting recognition, Pattern Recognition, 35: 229-244, 2002.

[16] J. Angulo and J. Serra. Automatic analysis of DNA microray images using mathematical morphology. Bioinformatics, vol 19, no 5, pp. 553-562, Mar 2003.

[17] B. El kessab, C. Daoui, B. Bouikhalene, M. Fakir, and K.Moro. Extraction Method of Handwritten Digit Recognition Tested on the MNIST Database, International Journal of Advanced Science and Technology Vol. 50, January, 2013.

[18] B. El kessab, C. Daoui, B. Bouikhalene, M. Fakir, and K.Moro. Handwritten Tifinagh Text Recognition using Neural Networks and Hidden Markov Models, International Journal of Computer Applications (0975 - 8887) Volume 75- No.18, August 2013. 
[19] M. Iwanowski and M. Swierez. Pattern Recognition Using Morphogical Class Distribution Functions and Classification Trees. Springer, pp. 143-154, 2011.

[20] J. Serra. Image Analysis and Mathematical Morphology. II: Theoretical Advances. Academic Press. London, 1988.

[21] A. Benouareh, A. Ennaji, and M. Sallami. Semi-continuous HMMs with explicit state duration for unconstrained Arabic word modeling and recognition. Pattern Recognition Letters archive. Volume 29 Issue 12, pages 1742-1752. September, 2008.

[22] R. S. Hegadi. Recognition of Printed Kannada Numerals based on Zoning Method. International Journal of Computer Applications (0975 - 8878) on National Conference of Advanced Computing and Communications - NCACC, April 2012.

[23] A. Hennig and N. Sherkat Exploiting zoning based on approximating splines in cursive script recognition. Pattern Recognition, Volume 35, Issue 2, February 2002, Pages 445- 454.

[24] D. Impedovo and G. Pirlo. Zoning methods for handwritten character recognition: A survey. Pattern Recognition, 47(3):969-981, 2014.

[25] S. Manipal, D.H Manjaiah, R. Bera, and H.N. Ashoka. Based Feature Extraction and Statistical Classification Technique for Kannada Handwritten Numeral Recognition, International Journal of Computer Science \& Engineering Technology (IJCSET) 2010.

[26] A. Rahman and B. Verma Effet of ensemble classifier composition on offline cursive character. Information Processing \& Management, Volume 49, Issue 4, July 2013, Pages 852-864.

[27] T. C.Mota and A. Thomé. One-Against-All-Based Multiclass SVM Strategies Applied to Vehicle Plate Character Recognition, IJCNN, 2009.

[28] G. Sinha and J. kumar. Arabic numeral Recognition Using SVM Classifier. International Journal of Emerging Research in Management \& Technology ISSN: 2278- 9359 (Volume-2, Issue-5), May 2013.

\section{Authors}

B. EL Kessab, received his Master's degree in 2009 from Faculty of Sciences and Technology University Sultan Moulay Slimane Beni Mellal Morocco, currently working on his Ph.D. in Information Processing and Decision Aids Laboratory at Sultan Moulay Slimane University. His current research interests include pattern recognition, image analysis, document processing and automatic processing of natural languages using hidden Markov models and neural networks.

C. Daoui, received his Ph.D degree on mathematics in 2002 from Mohamed V University Rabat Morocco. Currently is a professor in Faculty of Sciences and Technology, University Sultan Moulay Slimane Beni Mellal Morocco. His research topics are: the mathematics, operational research and pattern recognition.

B. Bouikhalene, received his $\mathrm{Ph} . \mathrm{D}$ degree on mathematics in 2001 and Master's degree on Science of Computer and Telecommunications in 2007 from the University Ibn Tofel Kenitra. Currently is a professor in the Sultan Moulay Slimane University Beni Mellal Morocco. His research topics are: the pattern recognition, artificial intelligence and mathematics and its applications.

R. Salouan, received his Master's degree in 2010 from Faculty of Sciences and Technology University Sultan Moulay Slimane Beni Mellal Morocco, currently working on his Ph. D in Sultan Moulay Slimane University. His current research interests include pattern recognition, image analysis, document processing and automatic processing of natural languages using hidden Markov models and neural networks. 
International Journal of Hybrid Information Technology Vol. 7, No. 6 (2014) 